\title{
Les petites « liturgies » politiques de l'islam au
}

\section{Gabon}

Ou comment lire les liens entre le politique et l'islam minoritaire

The Littles "Liturgies" of Political Islam in Gabon. Or how to Read the Links

between Politics and Minority Islam

\section{Doris Ehazouambela}

\section{OpenEdition}

\section{Journals}

Édition électronique

URL : http://journals.openedition.org/etudesafricaines/17120

DOI : 10.4000/etudesafricaines. 17120

ISSN : $1777-5353$

Éditeur

Éditions de l'EHESS

\section{Édition imprimée}

Date de publication : 1 juin 2012

Pagination : 665-686

ISBN : 978-2-7132-2349-5

ISSN : 0008-0055

Référence électronique

Doris Ehazouambela, " Les petites « liturgies » politiques de l'islam au Gabon », Cahiers d'études africaines [En ligne], 206-207 | 2012, mis en ligne le 01 juillet 2014, consulté le 30 avril 2019. URL http://journals.openedition.org/etudesafricaines/17120; DOI : 10.4000/etudesafricaines. 17120 


\title{
Les petites «liturgies » politiques de l'islam au Gabon
}

\author{
Ou comment lire les liens entre le politique \\ et l'islam minoritaire
}

On se propose, dans le présent texte, d'analyser les conditions de collusion de l'islam avec le champ politique au Gabon. Plus précisément, il s'agira d'examiner comment le champ religieux islamique, source de pouvoir matériel et symbolique, est utilisé par les membres éminents de la «société politique » gabonaise pour asseoir leur autorité et acquérir des moyens financiers ; cela dans un contexte où les imbrications entre religion et politique valent tant au plan local que national et international (Coulon 2002 : 19 ; Dozon 2008). Par conséquent, on interrogera la catégorie d'islam politique ou de politique par l'islam pour montrer comment le religieux est instrumentalisé par le politique afin d'occuper une position hégémonique (Bayart 1996). Dans cette perspective on examinera comment un certain monde musulman peut, dans une situation de minorité et d'immigration, se soumettre à un chef de l'État devenu lui-même calife. En effet le président Omar Bongo et les membres de la «société politique » se sont convertis à l'islam dans les années 1970, peut-être par conviction religieuse, mais plus sûrement parce qu'ils ont compris qu'il était susceptible de renforcer leur autorité symbolique et politique. Cela d'autant mieux que ces mêmes membres de la «société politique » cumulent les pratiques de l'islam, de la francmaçonnerie et de la Rose-Croix (Bongo 2001 : 118 ; Augé 2005 ; M'Bokolo 2009 : 35), cherchant, de la sorte, à superposer des apprentissages et chemins menant vers la connaissance spirituelle (Samson 2005 : 57), voire ésotérique $^{1}$ (Hamès 2007).

Mais avant d'examiner tout cela, il est nécessaire de retracer le cheminement historique de l'islam au Gabon.

1. De la même façon, il s'est constitué un marché local et international des «marabouts » que les hommes politiques notamment, ayant des moyens financiers importants, consultent à la veille ou à l'occasion de leurs multiples entreprises et compétitions électorales. 


\section{La présence musulmane au Gabon}

L'islam pénétra au Gabon à la fin du $\mathrm{XIX}^{\mathrm{e}}$ siècle et se développa durant la première moitié du $\mathrm{XX}^{\mathrm{e}}$, c'est-à-dire pendant la période coloniale. Les premiers musulmans furent des commerçants venus de leur propre initiative du Cameroun, du Tchad et de certaines colonies de l'Afrique de l'Ouest, à la recherche de débouchés commerciaux. Ensuite, les besoins en maind'œuvre nécessitèrent des auxiliaires pour la mise en valeur de la colonie. $\mathrm{Du}$ fait particulièrement aigu du sous-peuplement du territoire gabonais (Balandier 1982), les autorités coloniales durent faire venir des migrants également d'Afrique de l'Ouest dont beaucoup étaient musulmans. Ainsi, l'islam au Gabon fut largement contemporain de la colonisation française comme cela fut également le cas pour d'autres colonies de l'Afrique de l'Ouest (Triaud 2009) où le développement de l'économie monétaire fit une large place aux commerçants musulmans migrants (Brégand 1998).

Dans le cas plus particulier du Gabon, on distingue les «Haoussas » qui regroupaient un ensemble hétérogène de migrants venus d'eux-mêmes pour y exercer des activités de commerce, des autres migrants d'origine soudano-sahélienne qui étaient employés pour d'autres tâches au sein de l'administration coloniale en AEF. L'administration coloniale employa ces hommes comme commis de bureaux ou comme éléments de troupes de la milice coloniale 2 . De la sorte, il se constitua, dans la colonie du Gabon, une population musulmane minoritaire composée d' «Haoussas » et d' « Aofiens » (Bernault 1996).

Après l'indépendance, cette immigration de musulmans se poursuivit et s'amplifia avec l'effet conjugué du «boom pétrolier» des années 1970 et de l'urbanisation en Afrique subsaharienne (Piga 2003). Pour ses besoins en main-d'œuvre ${ }^{3}$, le Gabon recouru à nouveau à une population étrangère d'Afrique de l'Ouest spécialement destinée aux «grands travaux » publics, notamment la construction du chemin de fer. Des vagues d'immigrations musulmanes eurent lieu, venant de l'ensemble des pays d'Afrique de l'Ouest (Pourtier 1989b : 234-235). Le Gabon signa ainsi des conventions d'importation de main-d'œuvre avec le Sénégal, le Mali, la Guinée-Conakry et la Haute-Volta (aujourd'hui Burkina-Faso). Cette main-d'œuvre était fortement composée de musulmans. Par exemple, dans le cadre de ces accords, 5000 travailleurs sénégalais furent transférés à Libreville par la société

2. Il y avait en effet trois catégories de travailleurs coloniaux venus de l'AOF : agents administratifs, employés de compagnies concessionnaires et miliciens. Il faut également ajouter d'autres employés de maison.

3. Roland POURTIER (1989a) souligne qu'à son indépendance en 1960, le Gabon comptait 28000 habitants pour atteindre, en 1970, 950009 personnes recensées, soit une évolution de $51 \%$. Mais les chiffres sur le nombre réel d'habitants au Gabon ont toujours été considérés comme une question sensible politiquement, au point que certains recensements (1980 et 2003) n’ont jamais été rendus publics. Les résultats sont demeurés inaccessibles, comme de véritables secrets d'État. 
Dumez et furent installés dans un camp spécial à l'ouest de la capitale gabonaise dénommé «Lalala-Dakar » ${ }^{4}$ (Chouala 2004 : 99).

Sorte d'émirat africain, le Gabon ressembla ainsi à ses homologues du golfe Persique : un pays d'immigration de travail. Puis s'ajouta une immigration clandestine qui fit prospérer l'économie dite informelle. Finalement, compte tenu de l'importance prise par les immigrés d'Afrique de l'Ouest, spécialement sénégalais, ce sont les adeptes de deux confréries qui se distinguèrent tout particulièrement, en l'occurrence ceux de la Tijâniyya et ceux de la Mouridiyya. Si les seconds se regroupèrent autour de l'histoire symbolique de leur cheikh, Amadou Bamba, et de sa mémoire d'exil pendant sept ans au Gabon, les premiers s'appuyèrent sur l'importante contribution de leur confrérie à l'histoire de l'Afrique. Les deux groupes de confréries, de tendance soufie, furent (ou sont encore) respectivement dirigés par des ressortissants sénégalais, des cheikhs qui se succédèrent de la période coloniale jusqu'à ce jour. Plus tardivement, se constitua également l'Association des Haoussas du Gabon (AHG), création des descendants des premiers migrants « haoussas » honorant la mémoire de leurs parents. Ses objectifs sont essentiellement sociaux : encadrement et soutien scolaire de la jeunesse haoussa. Elle est, de ce point de vue, autant sociale que religieuse.

Encore faut-il ajouter qu'au cours des trois dernières décennies, l'islam ne fut pas seulement qu'une affaire de populations immigrées. Des phénomènes de conversion apparurent au sein de la population locale. Cette conversion des Gabonais fut le résultat du développement des interactions — relations d'amitié, d'alliances matrimoniales, d'échanges commerciaux entre commerçants musulmans et populations locales. Mais la «mutation » sociale de l'islam est liée aux conversions des Gabonais de la «société politique », ceux qu' on appelle les « Mamadou ». « Mamadou » désigne les hommes du haut de la stratification sociale gabonaise.

Ensuite, au cours de la même période, les conversions à l'islam furent aussi celles des gens ordinaires de la «société civile», qu'on nomme les « Makaya », c'est-à-dire les gens d'en bas de l'échelle sociale. Les conversions des «Makaya » sont à relier à l'intensification des interactions entre musulmans et non-musulmans au cours de la période de crise économique des années 1980, et à la mise en place, par le FMI et la Banque Mondiale, des programmes d'ajustement structurel qui s'en est suivie. Ce fut une période de précarisation des conditions de vie des « Makaya », où justement les sociabilités religieuses, notamment musulmanes, tentèrent d'offrir des nouvelles possibilités de sortie par la prise en charge ou l'encadrement social des fidèles. Ce fut donc à ce moment-là que des jeunes se convertirent, et que certains obtinrent des bourses pour aller étudier en Arabie Saoudite et en Égypte. Ces jeunes, pendant leur formation, reçurent des

4. Une partie de la zone est du «Quartier Lalala», dans le cinquième arrondissement de Libreville, fut entièrement occupée par des ressortissants sénégalais qui la renommèrent «Lalala-Dakar », en référence à leur capitale d'origine, Dakar. 
enseignements wahhabites. Certes, il n'y eut pas de conversions massives à l'islam, même si la communauté musulmane locale compte aujourd'hui des Gabonais partagés entre deux tendances, soufie et wahhabite. Les prêches de catégorie soufie représentent la majorité des musulmans au Gabon, tandis que la tendance wahhabite est incarnée par les «jeunes intellectuels » diplômés des instituts et universités d'Arabie Saoudite. Enfin, moins entreprenante religieusement, il existe également une minorité chiite constituée spécifiquement des immigrés syro-libanais.

\section{Une singularité de l'islam et la politique au Gabon}

L'acte de départ de la relation entre islam et politique au Gabon fut la conversion à l'islam d'Omar Bongo en septembre 1973. Au-delà de toute analyse sur sa conversion à l'islam, Omar Bongo indiqua que cet acte était le fait d'une nouvelle conviction religieuse, ce d'autant plus qu'après l'annonce de cette conversion, il accomplit son pèlerinage à La Mecque, devenant ainsi El Hadj Omar Bongo (Bongo 2001 : 112). Cette période fut alors le moment de conversion des « Mamadou » à l'islam, population incarnée par le président du Gabon, au pouvoir durant près d'un demi-siècle. De la sorte, se forma dans l'entourage de l'autorité politique gabonaise un groupe d'hommes (ministres, hauts fonctionnaires, officiers supérieurs d'armée, et autres) : celui des «Mamadou» convertis à l'islam.

Du coup, l'autorité politique s'imbriqua dans l'islam de deux manières. Tout d'abord, Omar Bongo devint le chef de tous les musulmans du pays en utilisant le dispositif religieux soufi local pour se transformer en un genre inédit de «calife général ». Ensuite, et dans la logique de ce qui précède, ce «calife général » fut, en même temps, «le président de la République, chef de l'État, et le premier des dirigeants politiques du pays $»^{5}$. Ainsi, les «liens » entre les politiques et l'islam prirent sens, sous forme du cumul des fonctions autour de la «figure » politique d'Omar Bongo qui incarnait un charisme particulier. Le pouvoir politique du défunt président de la République gabonaise revêtit alors une dimension religieuse avec sa conversion à l'islam, ce qui offre l'occasion d'analyser ses agissements d'homme politique en tant que musulman et, inversement, de musulman en tant qu'homme politique.

Dans ces petites « liturgies » politiques de l'islam il y eut, manifestement, une volonté exprimée par l'autorité politique d'investir l'ensemble des domaines d'intervention de l'islam au Gabon; ce que les musulmans eux-mêmes nomment « les affaires islamiques ». C'est effectivement du fait de la conversion de l'ancien président gabonais, ainsi que d'une partie de

5. J. BAUdouin (1998 : 187) considère la longévité politique du président Omar Bongo (au pouvoir pendant plus de 42 ans) comme l'une des situations caractéristiques (exemplaires) des régimes autoritaires. 
ses ministres, généraux, hauts fonctionnaires et autres, qu'un dispositif de « gestion », à la fois administrative et politique, fut instauré pour mettre en coalition et garantir la cohésion de toutes les composantes de l'islam local, regroupant les immigrés mourides, tidjanis, haoussas, et les convertis partagés entre soufisme et wahhabisme. Cette communauté musulmane locale, hétérogène et composée en grande partie d'une population immigrée, devait, effectivement, apprendre à être et à vivre unie : toutes conditions pour avoir une umma (communauté islamique) locale apaisée. L'ensemble des composantes de la communauté musulmane (les « Haoussas », Sénégalais, Maliens, Nigériens, Guinéens, et autres venus d'Afrique de l'Ouest) se devait d'observer le principe de solidarité entre «frères », caractéristique de la foi musulmane, contrôlée par l'État lui-même.

Ainsi, les pratiques politiques liées à l'islam au Gabon trouvent leur singularité par le fait qu'à partir des années 1970, une politique «volontariste » fut mise en place par des acteurs sociaux (politiques) convertis à l'islam, afin de tenter une sorte de vulgarisation de l'islam. Il s'agissait d'une forme de prosélytisme non offensive pour la propagation de la foi musulmane, consistant en une promotion des «affaires islamiques »dans le pays. Les «affaires islamiques » sont entendues ici comme l'ensemble des domaines sociopolitique et économique dans lesquels le lien avec l'islam est établi. Du fait de l'implication et de la place prise par les acteurs politiques convertis à l'islam (ou par ce que j'appelle la « société politique ») au sein de la communauté musulmane locale, celle-ci a acquis progressivement une visibilité dans « l'espace public religieux » (Holder 2009), notamment occupé par les Églises pentecôtistes et/ou évangéliques. Du coup, ces convertis à l'islam qui gouvernent en même temps l'État mirent en place des structures d'organisation sociale, afin de contenir et mieux gérer l'islam localement. La première structure fut le Commissariat politique aux relations extérieures chargé des affaires islamiques. Ce commissariat fut l'ancêtre de la seconde structure, du début des années 1990, l'actuel Conseil supérieur des affaires islamiques du Gabon (CSAIG). L'un des objectifs de ces différentes structures fut de rassembler les musulmans et de donner une voie officielle à l'umma locale. En ce sens, l'enjeu pour Omar Bongo fut de procéder à une sorte d'addition de sa politique extérieure aux «affaires islamiques », pour ainsi s'afficher avec les dirigeants des pays arabo-musulmans sur la scène locale et internationale.

\section{Que faire de I'appui de l'islam?}

Il s'agissait d'abord, pour la « société politique », de contribuer à la réalisation du projet «des grands travaux » de développement du pays initié, au début des années 1970, par la politique économique - dite de «Rénovation » — d'Omar Bongo, avec l'appui des pays du monde arabo-musulman. 
Pour ces «grands travaux », l'un des projets majeurs que les dirigeants gabonais entendaient réaliser avec le soutien financier de ces pays, fut le chemin de fer Transgabonais, image du progrès du pays, dont les travaux débutèrent le 7 avril 1972. Le désistement, en 1973, de la Banque Mondiale qui devait financer cet ambitieux projet qu'elle jugea trop onéreux, fut un événement conjoncturel qui embarrassa les dirigeants du Gabon. Cette conjoncture relégua à plus tard le projet de chemin de fer, ce qui n'était pas du goût du président Albert Bernard Bongo, tant le Transgabonais était au centre de sa politique territoriale. Il se voyait alors contraint de trouver de nouveaux partenaires pour financer son projet de chemin de fer.

Comme l'indique Roland Pourtier (1989b : 228) à propos de ce projet,

«C'est l'instrument par excellence de la construction de l'État. Il illustre de façon éclatante les liens entre espace et politique, entre économie et imaginaire spatial. Plus encore que la route, la voie ferrée affirme comme un emblème de sa puissance la prise de possession du sol par l'État [...]. Et entendre siffler le train en gare de Franceville a été le but poursuivi avec ténacité, presque jusqu'à l'obsession. "Je l'affirme devant vous : avec ou sans la Banque Mondiale, le Transgabonais se fera. Il se fera d'une façon ou d'une autre, s'il faut pactiser avec le diable, nous pactiserons avec le diable", déclarait le président de la République, Albert Bernard Bongo, le 25 février 1973 [...] le président du Gabon préférera pactiser avec les pays de l'islam riches de leur pétrole, et revient de la Mecque, El Hadj Omar Bongo.»

Dès l'instant qui suivit cette déclaration et en changeant son prénom d'Albert-Bernard en Omar, le président Bongo scellait le pacte, une alliance de coopération avec les pays arabes. Au moment où la Banque Mondiale refusa de financer le projet de chemin de fer Transgabonais, la conversion du président gabonais à l'islam apparaît donc comme une des voies salvatrices, voire stratégiques, en termes de ressources financières extérieures, pour contourner la difficulté du manque de bailleurs de fonds. Marquer son appartenance à l'islam lui permit de mettre à contribution les largesses financières des nouveaux partenaires du monde arabo-musulman. Les États arabes, d'ailleurs, tenaient à occuper la scène internationale afin de mettre en œuvre une véritable «politique africaine » (Otayek 2009: 21). De plus, René Otayek (1987 : 78) souligne que le guide libyen, Mohammar Kadhafi, dans sa volonté de cooptation des dirigeants africains, aurait également donné des pétrodollars favorisant la conversion de l'ancien président gabonais.

Dans ce contexte international marqué par la montée des pays arabomusulmans, notamment des pays comme la Libye, les relations entre Omar Bongo et Mohammar Kadhafi allaient bientôt prendre une dimension également religieuse, notamment lors de la construction d'un complexe islamique au Gabon. Mais ce projet, réalisé avec l'appui personnel du dirigeant libyen, prit une forme différente du projet d'origine, par la construction d'un lycée, le Lycée technique national Omar Bongo (LTNOB), et d'une mosquée, la « Mosquée d'Akébé », à Libreville, la capitale gabonaise. Des dires de la communauté musulmane, ce lycée fut un contre projet de celui qu'avait 
souhaité ériger au Gabon le guide libyen : faire construire, en plein pays d'Afrique centrale, un grand complexe islamique avec une université, des laboratoires, une bibliothèque et une résidence universitaire. Malgré le soutien financier de Kadhafi, Omar Bongo préféra toutefois accomplir son propre projet de construction d'un lycée technique au détriment de celui d'un complexe islamique souhaité par Kadhafi.

Si la conversion à l'islam d'Omar Bongo lui assura un soutien financier des dirigeants du monde arabo-musulman, elle lui permit également d'affirmer sa présence auprès de ces dirigeants et de faire adhérer son pays, le Gabon, aux organismes internationaux liés à ce monde arabo-musulman, parmi lesquels comptent l'Organisation de la conférence islamique (OCI) et l'Organisation des pays exportateurs de pétrole (OPEP).

Des « ressources» islamiques pour le Gabon

Ce fut donc à partir de septembre 1973, année de la conversion à l'islam du président Omar Bongo, que le Gabon siégea désormais à la table de presque toutes les organisations islamiques ; le Gabon adhère en premier à l'OPEP, le 19 novembre 1973, c'est-à-dire deux mois à peine après la conversion du président Bongo. Cette adhésion fut une stratégie, celle d'un rapide engagement, ce d'autant que le Gabon était devenu lui aussi un pays producteur de l'or noir et devait, à ce titre, bénéficier des pétrodollars. Le choix tactique d'adhésion à l'OPEP de la part d'Omar Bongo lui permettait alors de s'asseoir à la même table que d'autres dirigeants des pays du monde arabo-musulman et de rapprocher ainsi son pays le plus possible du lieu d'émission des pétrodollars nécessaires à la réalisation de ses projets nationaux de développement économique et social.

Le Gabon avait une population minoritairement musulmane contrairement au Nigeria par exemple. Son adhésion à l'OPEP n'avait pas pour but de faire bénéficier une large population musulmane des pétrodollars. En revanche, dans un contexte d'opacité dans la gestion des deniers publics, nul ne sait exactement combien d'argent le Gabon a obtenu de son entrée à l'OPEP qui, de plus, impliqua ses partenaires français, Shell et Elf Gabon, entreprises productrices du pétrole. Quoi qu'il en soit, il n'en demeure pas moins que cette adhésion ait contribué au financement des projets des « grands travaux » publics de l'État gabonais, notamment le Transgabonais qui fut l'un de ces projets majeurs. Le Gabon resta pendant plus de deux décennies membre de l'OPEP avant de démissionner, en 2002, du fait officiellement des taux élevés des contributions financières des États membres.

Dans cette même période des années 1970, le Gabon renforça sa participation à d'autres activités du monde musulman, comme celles entreprises par l'OCI à partir de 1974 (Sylla 1996 : 4). Cette organisation intergouvernementale, créée à la suite de l'incendie criminel le 21 août 1969 
de la Mosquée de al-Aksa à Jérusalem, un des lieux saints de l'islam ${ }^{6}$, finança les projets de développement au Gabon. Selon ses concepteurs, la vocation de l'oci est interétatique tout en ayant des objectifs plus ambitieux pour l'ensemble des pays membres, notamment dans les domaines de développement économique et social. Les dirigeants du Gabon espéraient ainsi faire profiter leur pays des objectifs de l'ocI en matière économique : l'éradication de la pauvreté, des maladies et de l'ignorance des populations.

Mais ce fut aussi le moyen pour Omar Bongo de s'accoler à un ensemble d'idéologies, prônées au cours des années 1970 par certains dirigeants des pays du monde arabo-musulman telles que la cessation de l'exploitation des pays en développement, la régulation des termes de l'échange entre pays développés et pays en développement (en matière de fourniture des matières premières), l'importation de produits manufacturés et de savoir-faire, la protection de la souveraineté des pays en développement et du contrôle inconditionné de leurs ressources naturelles, l'allègement de leurs difficultés occasionnées par la hausse des prix, etc.

Au sein de l'ocI, en 1977, le Gabon participa à la signature d'un accord de coopération économique, technique et commerciale, qui avait été élaboré par l'ensemble des États membres. Cet accord couvrit une gamme très étendue d'activités économiques qui reflétait les aspirations des États membres à ouvrer dans la solidarité (un des piliers de l'islam) dans le cadre d'une coopération économique et technique. Ce fut un programme ambitieux, conçu pour réaliser les objectifs de coopération entre États, par le truchement d'activités bilatérales et multilatérales. Il fut prévu, entre autres, le transfert de capitaux et d'investissements entre États, ainsi qu'une utilisation optimale du potentiel des États membres en matière de production alimentaire.

Suivant cette logique de promotion économique entre États et afin de faire valoir la présence du Gabon au sein de l'ocI, les dirigeants politiques décidèrent de la participation de leur pays :

« À la deuxième édition de la foire commerciale islamique inaugurée le 5 avril 1986 à Casablanca au Maroc, sous le mot d'ordre du "commerce inter-islamique au service du développement". M. Guy Nzouba Ndama7, alors Ministre délégué auprès du Ministre d'Etat, conseiller personnel du président de la république, Ministre de la consommation conduisait la délégation gabonaise » (Bignoumbe-Bi-Moussavou $1995: 34)$.

À cette rencontre économique, le Gabon obtint des promesses de financement des projets économiques, notamment dans le secteur de l'agroalimentaire. Il affirma également sa volonté d'aller jusqu'au bout des « affaires musulmanes » auprès du monde musulman. Effectivement, l'accumulation

6. Cette mosquée est considérée comme le troisième lieu saint de l'islam après La Mecque et Médine, tous les deux situés en Arabie Saoudite.

7. M. Guy Nzouba Ndama est l'actuel président de l'Assemblée nationale du Gabon, réélu pour un mandat de cinq ans, pour la quatrième fois. 
des «biens » symboliques et matériels fut au centre du pouvoir politique qu'incarna, tout au long de son règne, Omar Bongo dans sa direction du Gabon (Tonda 2005 : 158-161).

Comment accentuer la coopération avec des pays du monde arabomusulman

Outre la participation du Gabon aux organismes islamiques internationaux, ses dirigeants optèrent pour une politique de proximité, tous azimuts avec certains pays arabes, notamment l'Arabie Saoudite, le Koweït et le royaume du Maroc. Ainsi, ce fut dans cette visée de renforcement des liens de coopération bilatérale que le royaume d'Arabie Saoudite et le Gabon signèrent, en 1985, un protocole d'accords portant, entre autres, sur des projets de coopération touchant des domaines divers: l'installation au Gabon d'une banque des capitaux islamiques pour le financement de la construction des logements socio-économiques, l'ouverture d'une usine de production d'aliments pour enfants, la construction des quatre mosquées dans les capitales provinciales du pays (Makokou, Port Gentil, Franceville, et Oyem) ${ }^{8}$.

Sous un autre angle économique, la Banque Al Saudi d'un groupe d'hommes d'affaires saoudiens avait octroyé en 1984, au Conseil national du patronat gabonais (CNPG), une ligne de crédits de dix milliards de francs $\mathrm{CFA}^{9}$, forme d'emprunts sans intérêt. Les membres du CNPG utilisèrent à leur guise ces fonds islamiques pour la réalisation de leurs divers projets dont l'évaluation demeure aujourd'hui difficile à faire. De plus, cet argent ne bénéficia pas beaucoup au Gabon qui connut, malgré tout, une augmentation du chômage et de la pauvreté.

En fait, les rapports entre le Gabon et l'Arabie Saoudite étaient placés, comme le soulignait le quotidien gouvernemental L'Union, sous :

«Le label des relations de coopération bilatérales souhaitées par les plus hauts dirigeants des deux pays, le Roi Fahd et le Président El Hadj Omar Bongo Odimba ${ }^{10}$ [...]. L'Arabie Saoudite a réalisé de nombreux projets dans notre pays [...] il y a quelques années, le Royaume saoudien avait fortement contribué à la construction du chemin de fer ${ }^{11}$.

En effet, au cours d'une des visites des parlementaires saoudiens au Gabon, L'Union rajoute que :

8. L'Union, jeudi 7 mai 1985 , p. 5 .

9. L'Union, mardi 4 décembre 1984, p. 7.

10. Omar Bongo, selon un communiqué de son cabinet civil en date du 15 novembre 2003, avait rajouté le nom de son père Ondimba: il se nomme désormais El Hadj Omar Bongo-Ondimba.

11. L'Union, mercredi 9 mars 2005, p. 2. 
«Le Président du Conseil Consultatif (Saoudien) a donc insisté sur la nécessité pour les parlementaires des deux pays d'encourager les échanges économiques et commerciaux en œuvrant pour l'établissement des cadres juridiques propices encourageant des investissements ainsi que la venue des hommes d'affaires saoudiens en terre gabonaise $»^{12}$.

Par ailleurs, sous l'initiative, entre autres, de l'Arabie Saoudite, la Banque islamique de développement (BID), véritable cartel de pétrodollars directement financé par les fonds des pays arabo-musulmans, avait participé au financement d'autres projets de développement au Gabon avec des coûts jugés importants. Il s'est agi, par exemple, de la construction de l'Institut polytechnique de l'Université des sciences et techniques de Masuku (USTM) de Franceville dans le Haut-Ogooué13 ; de l'usine d'embouteillage d'eau minérale de Léconi (dans la même région); de l'extension du programme du palmier à huile par l'entreprise agroalimentaire AgroGabon à Lambaréné (sud-ouest); du financement pour un montant de 3478 milliards de francs CFA du projet de couverture globale du Gabon par les télécommunications, télévision, radio. Ce projet prévoyait également l'installation des centraux téléphoniques dans les localités du pays ${ }^{14}$. Pour le tout, le résultat fut assez probable à l'exception du programme du palmier à huile.

Il y eut, en plus des secteurs précités, d'autres domaines essentiellement culturels, comme l'Organisation islamique pour l'éducation, les sciences et la culture (ISESCO). L'ISESCO fut créée en mai 1980, après adoption par les États membres, à la onzième conférence des ministres des Affaires étrangères des pays islamiques tenue à Islamabad au Pakistan. C'est cette organisation qui avait signé le 5 septembre 1985 un accord de coopération avec le Centre international des civilisations bantu (CICIBA) dont le siège est à Libreville (Bignoumbe-Bi-Moussavou 1995 : 38). Le projet du CICIBA a pour objectif la promotion de ce qu'il est convenu d'appeler les cultures bantoues d'Afrique centrale.

Quid des relations " privilégiées » d'amitié entre le royaume du Maroc et la présidence de la République gabonaise?

La coopération entre le Gabon et le Maroc a aujourd'hui débordé du domaine purement économique en ajoutant le religieux islamique à l'échange. Effectivement en matière économique «le Royaume du Maroc pour sa part, premier partenaire commercial africain du Gabon exporte des fruits, des boîtes de conserve, etc. Il importe du bois et le pétrole [...] ${ }^{15}$. Les dirigeants des

12. Ibid.

13. C'est la région d'origine du président Omar Bongo.

14. L'Union, vendredi 7 décembre 1990, p. 6.

15. Ibid. 
deux pays voulurent donner un caractère particulier à cette coopération, grâce notamment à l'élan d'amitié entre Omar Bongo et l'ancien roi Hassan II. Cette amitié recherchée devait imprimer dans les représentations imaginaires locales une vision dite "sacrée » des relations entre les différents chefs. Si le caractère sacré du pouvoir est plus évident au Maroc, le pouvoir au Gabon a voulu en faire de même en se rapprochant de la royauté marocaine.

En dépit du fait que le roi Hassan II n'eut jamais visité le Gabon, son successeur héritier du trône, Mohammed vi le fit deux fois de suite, tandis que le président gabonais alla fréquemment au Maroc. Ces visites à répétition participaient de cette logique de «sacralité » des relations.

Ces propos de L'Union, lors d'une des visites du roi, sont révélateurs de cette particularité des relations:

«Le Roi du Maroc Mohammed VI a consacré le caractère privilégié de la coopération Maroco-gabonaise en entamant (hier) lundi 21 février 2005 une visite officielle dans notre pays, huit mois juste après celle, historique, de près d'une semaine effectuée en juin 2004 à Libreville [...] un accueil qui traduit l'exceptionnelle relation qui unit depuis des décennies, le Maroc et le Gabon, les peuples marocains et gabonais, ainsi que les liens d'amitié et de fraternité qu'entretiennent leurs dirigeants depuis feu Hassan II jusqu'à son successeur Mohamed VI [...]. Une visite d'amitié et de travail à forte tonalité politique, militaire, économique et humanitaire ${ }^{16}$.

Ces écrits du quotidien d'informations gouvernementales traduisent effectivement le caractère «sacré » qui entoure les relations religieuses, politiques et économiques entre le Gabon et le Maroc. Il s'agit plus précisément « de marquer les liens qui unissent le feu président gabonais et la famille royale, liens renforcés notamment sur le plan religieux $»^{17}$. Ainsi, lors de la dernière visite en date du roi du Maroc, fut organisée, en marge des rencontres officielles, une grande prière du vendredi à la Mosquée Hassan II, lieu symbolisant ces liens religieux. Le roi Mohammed VI et El Hadj Omar Bongo-Odimba, le «calife général » des musulmans du Gabon, étaient évidemment présents. Cette prière du vendredi, à portée symbolique et religieuse significative, avait été l'occasion pour le roi Mohamed vi de remettre un don de milliers d'exemplaires du Coran aux musulmans du Gabon, à commencer par le premier d'entre eux, leur «calife général ».

C'est également à l'occasion de cette visite que fut signé un accord en matière économique entre le gouvernement gabonais et des entreprises marocaines du bâtiment pour la réalisation d'un projet de construction de logements dans la zone périurbaine d'Agondjé, à une dizaine de kilomètres au nord de la commune de Libreville.

16. L'Union, mardi 22 février 2005, p. 2.

17. Ibid. 
Comment « gérer » la communauté musulmane locale

Dans cette implication du politique dans les « affaires islamiques » au Gabon, la gestion interne de la communauté musulmane ne fut pas en reste. Le premier organe de gestion de l'islam, mis en place par la «société politique », fut le Commissariat politique aux relations extérieures chargé des affaires islamiques. Ce commissariat avait été dirigé pendant près d'une décennie par l'un des membres de la «société politique », Barthélemy Moapa Beotsa, devenu El Hadj Mohammed Moapa Beotsa après sa conversion à l'islam en 1974. Ce fut l'une des personnalités du régime du parti unique instauré dès 1968 par le président Bongo. Avant de diriger le commissariat, Moapa Beotsa fut successivement, à partir de 1967 jusqu'en 1976, nommé ambassadeur du Gabon en Belgique, en Israël, en Espagne, au Congo Brazzaville, en Iran, puis, en 1979, il fut nommé ambassadeur itinérant à la présidence de la République. C'est finalement en 1980 qu'il fut nommé commissaire politique aux relations extérieures et membre du Bureau politique du parti démocratique gabonais (PDG) (très haute fonction au sein de ce parti jadis unique). Pendant six ans, de 1984 à 1990, il occupa ses fonctions de commissaire politique aux relations extérieures chargé des affaires islamiques, puis il devint président du Haut Conseil des musulmans du Gabon. À ce moment-là, il fut alors à la fois un représentant de l'autorité politique et islamique.

Avec le Haut Conseil des musulmans du Gabon, Mohammed Moapa Beotsa, sur instruction du président de la République, œuvra auprès de la Ligue islamique mondiale (LIM) pour la mise sur pied des mécanismes de diffusion et de propagation de l'islam sunnite au Gabon. Il fut amené, notamment avec l'appui financier de l'Arabie Saoudite, à concevoir le projet de construction des mosquées dans l'ensemble du territoire gabonais.

L'existence d'un commissariat politique aux relations extérieures chargé des «affaires islamiques » responsable de la construction des mosquées, permet de saisir la nature des rapports entre l'islam et le système politique au Gabon. En effet, le système politique gabonais qui fonctionnait, sous le règne d'Omar Bongo, avec une concentration des pouvoirs (Nze-Nguema 1998 : 123), devait s'assurer également de la gestion des « affaires islamiques ».

De fait, l'ensemble des «affaires islamiques » devenait un domaine réservé de la politique d'Omar Bongo, et Moapa Beotsa s'employa à recommander «aux musulmans d'être toujours loyaux et honnêtes comme le commande le Coran, de respecter les lois réglementant l'immigration dans le pays en étant en règle avec les pouvoirs publics et les forces de sécurité, afin de conserver la cohésion entre fidèles musulmans présents au Gabon ». Ainsi, le commissariat fut une forme «d'appareil religieux » (musulman) d'État qui entendit poser des œuvres en faveur de la communauté musulmane et vulgariser la foi musulmane (surtout changer sa perception) auprès 
des autochtones non musulmans ${ }^{18}$. Mais ce commissariat avait manifestement un objectif également politique : celui de contrôler l'immigration. En effet, le commissariat avait conçu un service spécial de délivrance de titres de séjours aux immigrés musulmans en collaboration avec les services du ministère de l'Intérieur. Les immigrés, surtout les clandestins, devaient donc s'adresser directement au commissariat qui constituait avec eux les dossiers de demandes de titres de séjours avant de les transmettre aux services du ministère de l'Intérieur. Ils complétaient ainsi leurs statistiques en matière d'immigration. Dans la plupart des cas, les dossiers passant par cette procédure aboutissaient à une régularisation, bien que celle-ci devenait plus onéreuse pour le migrant qui payait deux à trois fois plus le montant d'un titre de séjour $^{19}$. Ce fut également le commissariat qui organisa le voyage de pèlerinage à La Mecque pour les musulmans du Gabon. Toutes les demandes de pèlerinage passaient par le commissariat qui se chargeait, à la fin du processus, de publier la liste des pèlerins.

Naissance du Conseil supérieur des affaires islamiques du Gabon (CSAIG) : contrôle de l'islam local

Le Commissariat politique aux relations extérieures chargé des affaires islamiques fonctionna jusqu'au début des années 1990. Il avait pour objectif de «surveiller » l'ensemble des « affaires islamiques », mais il revêtit, au fil du temps, un caractère lucratif, recevant, entre autres, des dons et legs venus directement des pays du monde arabo-musulman ou de la présidence de la République gabonaise. Or, à mesure des années passant, la gestion des « affaires islamiques » par le commissaire Moapa Beotsa fut contestée par certains de ses coreligionnaires pour cause notamment de mauvaise gestion : on l'accusa de détournements. L'autre grief contre lui fut sa proximité avec les diplomates iraniens qui voulurent favoriser les enseignements de l'islam chiite au sein des mosquées. Pour mettre fin aux tensions persistantes entre prétendants à la nouvelle gestion, Omar Bongo tenta de reprendre les choses en main.

Autrement dit, comme un instrument aux mains de l'État pour contrôler les «affaires islamiques » locales (délivrance de titres de séjours, dons et legs arabo-musulmans, nominations d'imams, organisations du voyage de pèlerinage, etc.), la présidence de la gestion «des affaires islamiques » fut

18. Au moment de la conversion à l'islam des membres de la «société politique », cette religion est perçue avec des préjugés négatifs par des populations gabonaises qui l'assimilaient à une pratique ésotérique des «marabouts».

19. La délivrance ou l'obtention du titre de séjour est une question très sensible à aborder avec les responsables du CSAIG ou même avec les principaux concernés, les migrants. À titre indicatif, au Gabon, le coût moyen d'un titre de séjour est de 250000 F CFA $(380 €)$. 
confiée à M. Ali Bongo, fils du président Bongo, lui-même converti à l'islam en même temps que son père. Mais le commissariat, devenu par la suite Conseil des musulmans du Gabon (CMG), se mua en Conseil supérieur des affaires islamiques du Gabon (CSAIG). C'est le CSAIG qui amorça une phase importante dans la direction des « affaires islamiques », devenant depuis lors l'instance représentative et de direction de l'ensemble des composantes des musulmans du Gabon. Il fut placé sous l'autorité politique et morale d'Omar Bongo, président de la République et «calife général».

Selon ses concepteurs, le CSAIG est l'instance suprême de l'organisation islamique nationale qui fonctionne comme une institution de l'État, et a pour but de représenter la communauté musulmane du Gabon, de défendre sa dignité ainsi que ses intérêts légitimes. En tant qu'unique interlocuteur officiel des pouvoirs publics, le CSAIG coopère et traite avec les organisations internationales islamiques. Il a aussi pour mission de se préoccuper de l'image, de la défense des intérêts sociaux des musulmans et de la valorisation de l'islam dans l'opinion publique. Le CSAIG doit, pour ses dirigeants, s'ériger en un organe direct de contrôle politique et d'administration des «affaires islamiques» dans les domaines les plus divers de la vie de la communauté musulmane du Gabon. Dès lors, pour parachever cette vision de la gestion et la rendre plus coercitive, il fallut trouver le moyen d'obtenir le consensus de toutes les sensibilités de la foi musulmane. Pour ce faire, l'idée d'organiser à Libreville un «congrès » des musulmans du Gabon vit le jour.

Le 8 mai 2004, fut organisé, à la Cité de la démocratie de la capitale gabonaise, un « congrès » des musulmans où l'ensemble des acteurs et représentants des communautés et des associations à caractère musulman, avait répondu présent. Tout avait été préparé en amont au sein des mosquées (informations, distributions des dons, etc.) pour convaincre les musulmans de l'importance de leur participation à cet événement inédit. L'acte majeur et significatif pour l'ensemble des musulmans fut la signature de « la charte de la communauté musulmane du Gabon ». C'est un document d'une trentaine de pages qui paraît «complet» pour emmener l'adhésion de tous les musulmans locaux. Par cette charte, la question de la présidence du CSAIG, instance représentative de la communauté musulmane, fut réglée d'une manière définitive. Dans la mesure où le président de la République, Omar Bongo-Ondimba, avait été réaffirmé au rang symbolique de "calife général », il fut choisi comme la seule autorité physique et morale désignée par tous pour nommer ou démettre le président du CSAIG (dispositions stipulées au titre 1 de ladite charte).

De fait, on comprend que le résultat de ce «congrès » des musulmans allait de pair avec l'idée qu'il fallait, pour les responsables du CSAIG, l'établissement d'un consensus entre les différentes composantes de la communauté musulmane locale très hétérogène. Ce consensus reposa alors sur l'adhésion de tous les musulmans à des préceptes tels que l'instauration 
d'un «califat » et le contrôle social des dirigeants du CSAIG. C'est cette stratégie de persuasion qui conduisit à instaurer la cohésion entre les mourides, les tidjanis, les «arabisants », les présidents d'associations musulmanes, et autres.

Toutefois, des critiques furent adressées au «calife général », venant du côté des « jeunes intellectuels » musulmans (les arabisants). Il s'agissait de jeunes Gabonais convertis, ayant vécu dans les pays du monde arabomusulman pour suivre leurs études islamiques. Nombreux d'entre eux sortaient (sortent encore) des écoles et universités d'Arabie Saoudite et d'Égypte. Ils contestaient, en particulier, la désignation d'un «calife général » des musulmans au Gabon qu'ils jugeaient insensé dans le contexte gabonais. Ces «jeunes intellectuels» musulmans remirent en cause le fait qu'Omar Bongo était, en même temps, chef franc-maçon et «calife général » des musulmans. Dans leur perception, l'univers de la franc-maçonnerie (Bongo 2001 : 117-118) était incompatible avec celui de l'islam. Ces « jeunes intellectuels » musulmans gabonais prônaient un islam normatif et légaliste, selon le Coran, proche de l'idéologie wahhabite qu'ils avaient reçue pendant leurs études en Arabie Saoudite. Ainsi, grâce à leur savoir théologique, voire doctrinal, et conscients de leur connaissance littéraire de la langue arabe, et donc du Coran, ces «jeunes intellectuels» musulmans s'insurgèrent contre la pensée et les agissements des responsables du CSAIG et des vieux imams jugés rétrogrades. Aussi refusèrent-ils, en tant que wahhabites, de se plier au commandement d'un «califat». Mais, leur position peu reprise ou mise en minorité au sein de la communauté musulmane, les obligea à s'accommoder de la volonté de ceux qui dirigeaient, à la fois les «affaires islamiques » et l'État. En effet, l'État au Gabon, comme au Bénin, garantit la «bonne cohabitation » entre les religions (Brégand 2006) et entre leurs pratiquants. Plus spécifiquement l'État favorise la cohésion entre les différentes communautés, les pratiquants et les tendances des confréries au sein de la communauté musulmane locale.

Dans le domaine social de la gestion de la communauté musulmane locale, le CSAIG organise, depuis lors, le pèlerinage à La Mecque, fixe les modalités et le nombre de pèlerins chaque année. C'est aussi à travers les services du CSAIG que s'effectue la distribution des biens, des dons et legs dans les différentes mosquées aux musulmans, surtout pendant le mois de Ramadan, et les autres fêtes religieuses. Le CSAIG nomme certains imams dans l'ensemble des mosquées qui sont sous sa tutelle ${ }^{20}$. Existe également un service «spécial » de délivrance des titres de séjours aux résidents étrangers.

20. Toutes les mosquées du Gabon ne sont pas sous la tutelle du CSAIG. En effet, le CSAIG n'a sous sa tutelle que les mosquées dites historiques ou celles qui ont été construites avec l'appui des pays arabo-musulmans. D'autres mosquées dites de particuliers ne lui sont pas soumises en termes de nomination d'imams, par exemple. Ce genre de mosquées de particuliers est souvent la propriété de certains commerçants ou/et notables musulmans. 
La place du «calife général»

Si régler, une fois pour toute, la «question» de la direction des «affaires islamiques » revêt un enjeu majeur pour les membres de la «société politique » convertis à l'islam, cette question a également l'objectif de donner plus d'autorité au président Omar Bongo au sein de la communauté musulmane locale : celle hautement symbolique de «calife général».

Les charges confiées au "calife général », ayant des fonctions honorifiques, puisent dans un fond religieux connu des adeptes de Muhammad. C'est à lui, en tant que chef des musulmans, de procéder, à chaque fête de l'Aid El Kebir, à l'acte symbolique de l'immolation du premier mouton. Selon la conception de certains musulmans gabonais, "le calife est le commandeur des croyants [...], chef religieux garant du respect de la loi, il veille à ce que les droits coraniques de Dieu et des hommes soient observés sur terre. Le calife a la charge d'administrer les communautés; ce qui le hausse au rang de chef politique» (De Saingre 1977: 68). Omar Bongo, à la fois chef politique et chef religieux devint ainsi régulateur et garant de la cohésion du «tissu social » gabonais islamique. Pour cela, sa personne fut considérée comme «sacrée » par les uns et « honorifique » par les autres, pour qui, selon Jean-Paul Charney (1994: 611), le calife :

« [...] est un mythe vivant, vénéré et respecté. Ses moindres faits et gestes peuvent susciter des mouvements de force extraordinaire et des scènes d'hystérie. Ses déplacements dans la ville ou ses régions en dehors, entrent dans l'histoire [...] à l'occasion des fêtes religieuses et des pèlerinages. Comme "vicaire de Dieu sur terre" qui, dans le cadre d'un islam institutionnalisé symbolise le pouvoir suprême. »

De plus, le pouvoir du calife se singularise par la conjugaison (ou non) des différentes formes de légitimation décrites par Max Weber (1971), comme « rationnelle-légale » (l'autorité administrative), «traditionnelle » (de façon héréditaire) et « charismatique ». C'est surtout cette dernière qui fonde l'organisation du pouvoir maraboutique, dont le calife est l'autorité «suprême ». Même si dans le cas du Gabon, la mise en scène du «califat » ne donne pas à voir tous ces attributs, cette fonction de calife semble faire écho à l'objectif qu'Omar Bongo s'était fixé : entouré des « grands » marabouts de son pays, voire de ceux issus du «marché » du maraboutage international, il désirait détenir un pouvoir et un charisme certains.

Mise en scène du «califat »

Le fait d'être président et «calife » offrait à Omar Bongo des soutiens très larges. Ainsi lors de ses apparitions pendant des occasions religieuses (fêtes religieuses, prières du vendredi), notamment à la Mosquée Hassan II, toute une foule non seulement protocolaire, mais aussi et surtout musulmane se 
mobilisait. Les fidèles musulmans amassés autour et à l'intérieur de la mosquée attendaient impatiemment et parfois avec une certaine curiosité l'apparition de leur «calife».

De plus, les attributs conférés au «calife » trouvent leurs référents originels en latence dans la lignée du prénom musulman d'Omar que porte le «calife général » du Gabon. En effet, Omar, dans l'histoire de l'islam, est un personnage «converti, conquérant et organisateur» (De Saingre 1977). Si Muhammad est le législateur inspiré, Omar est par excellence l'artisan de l'expansion islamique. Tout cet imaginaire autour du prénom d'Omar ${ }^{21}$ traverse les représentations des musulmans et cela conduit à la croyance qu'Omar Bongo, président du Gabon, aurait été choisi par une sorte «d'élection divine » (d'ordre islamique soufi) - la baraka — pour devenir le «calife général» de la communauté musulmane du Gabon. De ce fait, son autorité devait s'exprimer en tant que chef de l'État, chef suprême des armées, chef de la magistrature suprême, et «calife général». Dans cette description des choses, sa direction des «affaires islamiques » venait justement de la logique du crédit que lui accordaient les musulmans en tant que chef «suprême ». L'idée était que, selon une conception soufie, ce cumul d'attributs (Dozon 1995) faisait de lui un personnage exceptionnel pour conduire les «affaires» du pays.

Ce faisant, toute action du président Bongo à l'endroit des musulmans était légitimée par cette double casquette de religieux et de chef de l'État. Cela fut illustré lors de la dernière apparition d'Omar Bongo, accompagné du roi Mohammed VI à la Mosquée Hassan II, la plus grande mosquée de Libreville, au cours de la prière du vendredi 25 février 2005 .

Le roi du Maroc était revenu, huit mois après son voyage historique de juin 2004 à Libreville, en visite officielle au Gabon. Cette prière du vendredi, diffusée par les deux chaines de télévisions publiques RTG1 et RTG2, fut un acte qui authentifia la symbolique religieuse du rapport entre le pouvoir chérifien et celui du président Bongo. C'est ainsi qu'au cours de cet événement unique, où le «calife général » se trouva aux côtés de sa majesté le roi du Maroc, la communauté musulmane, dans toutes ses composantes, notamment celles qui avaient signé auparavant la charte, le reconnut en cette double qualité de «chef» religieux et de chef de l'État. Tout cela participait à la compréhension d'un imaginaire populaire qui symbolisa le pouvoir politique d'Omar Bongo comme un cumul des «pouvoirs », médiation essentielle qui assoit la violence symbolique élaborée et administrée (Tonda 2005) pour l'exercice du pouvoir.

Cette reconnaissance s'est révélée au cours du dernier acte en date, posé par les musulmans du Gabon à l'endroit de leur «calife général », lors de l'hommage qu'ils lui ont rendu pendant des obsèques nationales organisées

21. Omar fut également un personnage qui marqua considérablement l'expansion de l'islam en Afrique de l'Ouest. On peut lire utilement à ce sujet D. ROBINSON (1985). 
après son décès, le 8 juin 2009, à Barcelone. Tout comme le reste du pays, la communauté musulmane s'était mobilisée pour rendre un hommage digne et mérité à son «calife général», exprimant ainsi aux yeux de l'opinion que le président défunt était aussi leur chef. Au cours des obsèques nationales d'Omar Bongo, plusieurs hommages lui ont été rendus par différents groupes sociaux (groupes des francs-maçons, des hommes de lettres, des politiciens amis, et autres) défilant devant la dépouille du président.

Cet hommage rendu au «calife général » des musulmans ressembla à ce que Pierre Bourdieu (1994: 210) avait appelé la violence symbolique qui « repose sur l'accord entre les structures constitutives de l'habitus des dominés et la structure de la relation de dominant à laquelle ils (ou elles) s'appliquent : le dominé perçoit le dominant à travers des catégories que la relation de domination a produites et qui, de ce fait, sont conformes aux intérêts du dominant ». En fait, l'émotion suscitée par la mort d'El Hadj Omar Bongo-Ondimba, survenue après celle de son épouse Édith-Lucie Bongo le 14 mars 2009 (un peu moins de trois mois avant), avait contribué à «l'hommage orchestré des Gabonais à "papa Bongo" »" 22 . Ce moment d'émotion nationale évacua, même pour un instant, toutes les exaspérations et critiques des populations envers le défunt président et son régime. Les populations, en général, les musulmans en particulier, et les jeunes générations habituées au président Omar Bongo redoutaient le vide que sa mort avait créé et les plongeait dans les incertitudes de l'histoire (politique) du pays.

Avec la succession d'El Hadj Ali Bongo-Ondimba à El Hadj Omar BongoOndimba aux fonctions de président de la République gabonaise, le couple islam et politique au Gabon a encore de beaux jours devant lui. En effet, à l'issue des assises du deuxième congrès des musulmans, organisé le 13 février 2010 à Libreville, les musulmans du Gabon ont, comme ils l'avaient fait pour Omar Bongo, désigné Ali Bongo Raïs comme chef, président de tous les croyants de la foi musulmane. Par cette fonction, il devient ainsi le garant de la cohésion entre musulmans, prenant soin, à son tour, de désigner les nouveaux responsables du CSAIG, tout comme l'avait procédé son père El Hadj Omar Bongo-Ondimba en son temps.

Centre d'études africaines, EHESS, Paris.

22. Libération, mardi 16 juin 2009. Voir également J. TondA (2009). 


\section{BIBLIOGRAPHIE}

Assoumou Mвомвеy, T.

1989 L'évolution de l'islam au Gabon de 1973 à nos jours, Mémoire de maîtrise, Libreville, UOB, FLSH.

Augé, A.-E.

2005 Le recrutement des élites politiques en Afrique subsaharienne. Une sociologie du pouvoir au Gabon, Paris, L'Harmattan.

BALANDIER, G.

1982 Sociologie actuelle de l'Afrique noire. Dynamique sociale en Afrique centrale, Paris, PUF.

BAUdouin, J.

1998 Introduction à la sociologie politique, Paris, Éditions du Seuil.

BAYART, J.-F.

1996 L'État en Afrique. La politique du ventre, Paris, Fayard.

Bernault, F.

1996 Démocraties ambigües en Afrique Centrale : Congo-Brazzaville, Gabon, Paris, Karthala.

Bignoumbe-Bi-Moussavou, I.

1995 L’islam au Gabon, Libreville, éd. Lumière/médias.

BONGO, O.

2001 Blanc comme Nègre, Paris, Grasset.

Bourdieu, P.

1994 Raisons pratiques. Sur la théorie de l'action, Paris, Éditions du Seuil.

BRÉGAND, D.

1998 Le commerce caravanier et les relations sociales au Bénin, Paris, L'Harmattan.

2006 «La Ahmadiyya au Bénin», Archives de sciences sociales des religions, $135: 73-90$.

Charney, J.-P.

1994 La sociologie religieuse de l'islam, Paris, Hachette.

Chelhod, J.

1983 Introduction à la sociologie de l'islam, Paris, éd. Bessous.

Chouala, Y. A.

2004 «L'installation des Camerounais au Gabon et en Guinée-Équatoriale : les dynamiques originales d'exportation d'État d'origine », in L. SindJoun (dir.), Etat, individus et réseaux dans les migrations africaines, Paris, Karthala : 93114. 
Coulon, C.

2002 «Les nouvelles voies de l'umma africaine», in L'Afrique politique, Islams d'Afrique : entre le local et le global, Paris, Karthala-CEAN : 19-29.

CuoQ, J.-M.

1975 Les musulmans en Afrique, Paris, Maisonneuve et Héros.

De Saingre, G.

1977 L'islamisme, Paris, éd. Seghers.

Dozon, J.-P.

1995 La cause des prophètes. Politique et religion en Afrique contemporaine, Paris, Éditions du Seuil.

2008 L'Afrique à Dieu et à Diable. États, ethnies et religion, Paris, Ellipses.

Gaborieau, M. \& Zeghal, M.

2004 «Les autorités religieuses en islam », Archives de sciences sociales des religions, $925: 5-22$.

Germais, E.

2007 L'Afrique du Sud musulmane. Histoire des relations entre Indiens et Malais $d u$ Cap, Paris, Karthala.

GiLquin, M.

2010 Atlas des minorités musulmanes en Asie méridionale et orientale, Paris, Éditions du CNRS.

GoMeZ-PÉREZ, M. (DIR.)

2005 L'islam politique au sud du Sahara. Identités, discours et enjeux, Paris, Karthala.

HAMÈs, C. (DIR.)

2007 Coran et talismans. Textes et pratiques magiques en milieu musulman, Paris, Karthala.

HOLDER, G.

2009 «Vers un espace public religieux : pour une lecture contemporaine des enjeux politiques de l'islam en Afrique », in G. HoLDER (dir.), L'islam, nouvel espace public en Afrique, Paris, Karthala : 5-36.

M'BoкоLо, E.

2009 Médiations africaines. Omar Bongo et les défis diplomatiques d'un continent, Paris, L'Archipel.

NZENG, A.

1985 Essai sur l'islam à Libreville de 1900 à nos jours, Mémoire de maîtrise, Libreville, UOB, FLSM.

Nze-Nguema, F.-P.

1998 L'État au Gabon de 1929 à 1990 : le partage institutionnel du pouvoir, Paris, L'Harmattan. 
OTAYEK, R.

1987 La politique africaine de la Libye (1969-1985), Paris, Karthala.

Otayek, R. \& Soares, B.

2009 «Islam et politique en Afrique », in R. OtAYek \& B. SoARes (dir.), Islam, État et société en Afrique, Paris, Karthala-CEAN.

PigA, A.

2003 Islam et villes en Afrique au sud du Sahara. Entre soufisme et fondamentalisme, Paris, Karthala.

POURTIER, R.

1989a Le Gabon : espace-histoire-société, t. 1, Paris, L'Harmattan.

1989b Le Gabon: État et développement, t. 2, Paris, L'Harmattan.

RoBinson, D.

1985 The Jihad of Al Hajj Omar, Clerendon, Clerendon University Press.

Rossantaga-Rignault, G.

2005 «Au titre des mesures individuelles... Petit catéchiste des liturgies politiques gabonaises », Rupture-Solidarité, 6, Paris, Karthala : 11-31.

Russ, J.

1994 Les théories du pouvoir, Paris, Librairie générale française.

SAMSON, F.

2005 Les marabouts de l'islam politiques. Le Dahiratoul Moustarchidina Wal Moustarchidaty, un mouvement néo-confrérique sénégalais, Paris, Karthala.

Sylla, A.

1996 Les relations entre le Gabon et l'organisation de la conférence islamique de 1974 à nos jours, Rapport de licence, Libreville, UOB, FLSM.

TONDA, J.

2005 Le souverain moderne. Le corps du pouvoir en Afrique centrale (Congo, Gabon), Paris, Karthala.

2009 « Omar Bongo-Ondimba. Paradigme du pouvoir postcolonial», Politique Africaine, 114 : 127-139.

TRIAUD, J.-L.

2009 «Une laïcité coloniale. L'administration française et l'islam en Afrique de l'Ouest (1860-1960)», in C. Peyrard (dir.), Politique, religion et laïcité, PUP : 121-143.

TRIAUd, J.-L. \& Robinson, D. (DIR.)

2000 La Tijâniyya. Une confrérie musulmane à la conquête de l'Afrique, Paris, Karthala.

WEBER, M.

1971 Économie et société, t. 1, Paris, Plon. 


\section{RÉSUMÉ}

Ce texte analyse les liens qui existent au Gabon entre le pouvoir politique et l'islam minoritaire. D'une religion introduite par des vagues $d^{\prime} i m m i g r a t i o n s ~ s u c c e s s i v e s$, I'islam connaît sa conjoncture au début des années 1970, avec notamment la conversion des "Mamadou», des hommes politiques autour de la figure tutélaire de l'ancien président Omar Bongo-Ondimba. Par une stratégie politique d'accumulation des pouvoirs, El Hadj Omar Bongo-Ondimba devient le « calife général » des musulmans de son pays. Ce positionnement donne à son pays accès à des pétrodollars dont les pays du monde arabo-musulman sont pourvoyeurs. Mais aussi à travers la mise en visibilité de l'islam sur le plan local, le Gabon développe une coopération multilatérale avec les mondes musulmans. De plus, I'organisation de I'islam en Conseil supérieur des affaires islamiques du Gabon (CSAIG) permet à l'État d'organiser la communauté musulmane et de conserver ainsi la cohésion sociale de son hétérogénéité. Ce texte tente de répondre au questionnement suivant : quel type de rapports le politique entretient avec l'islam au plan local et international ? Comment se structure l'umma gabonaise?

\section{ABSTRACT}

The Littles "Liturgies" of Political Islam in Gabon. Or how to Read the Links between Politics and Minority Islam. - This text analyzes the links between political power and minority Islam in Gabon. Islam, a religion introduced by successive waves of immigration, makes an appearance in the beginning of the 1970s, with the conversions of the "Mamadou", or politicians around the tutelary figure of former president Omar Bongo-Ondimba. Through a political strategy of accumulation of power, El Hadj Omar Bongo-Ondimba became the "calife général" of the Muslims in his country. Through this national emphasis on Islam, Gabon developed a multilateral cooperation with the rest of the Muslim world, as well as permitting the country access ton petrodollars supplied by the Arab/Muslim states. The Conseil supérieur des affaires islamiques du Gabon (CSAIG) allows the state to organize the Muslim community as well as preserving social cohesion and heterogeneity. This article addresses the following questions: What types of relationship does politics have with Islam on the national and international levels? How is the Gabonese umma structured?

Mots-clés/Keywords : Gabon, calife général, conversion, cumul, islam, pétrodollars, raïs, société politique, umma locale/Gabon, general calife, conversion, accumulation, islam, petrodollars, raïs, political society, local umma. 